\title{
A SVR-Based Multimedia Streaming Dissemination System for Vehicular Networks
}

\author{
Chenn-Jung Huang, Kai-Wen Hu, Heng-Ming Chen, Yu-Wu Wang, Jui-Jiun Jian, and Sheng-Yuan \\ Chien
}

\begin{abstract}
An adaptive seamless streaming dissemination system for vehicular networks is presented in this work. An adaptive streaming system is established at each local server to prefetch and buffer stream data. The adaptive streaming system computes the parts of prefetched stream data for each user and stores them temporarily at the local server, based on current situation of the users and the environments that they are located at. Thus, users can download the prefetched stream data from the local servers instead of from Internet directly, such that the video playing problem caused by network congestion can be avoided. Several techniques such as stream data prefetching, stream data forwarding, and adaptive dynamic decoding, were utilized for enhancing the adaptability of different users and environments and achieving the best transmission efficiency. Support vector regression (SVR) is utilized to determine if a roadside base station (BS) or a vehicle can be chosen to transfer stream data for users. Considering the uneven deployment of BSs and vehicles, the bandwidth reservation mechanism for premium user was proposed to ensure the QoS of stream data premium user received. A series of simulations were conducted, the experimental results verify the effectiveness and feasibility of the proposed work.
\end{abstract}

Index Terms-Adaptable dynamic decoding, adaptable streaming service dissemination, bandwidth reservation, stream data prefetch, support vector regression, vehicular networks.

\section{INTRODUCTION}

With advanced network technology, people can share digital media information through Internet, in which vehicular networks are respected to be well developed in the future. Through vehicular networks, vehicles can exchange real time information to avoid accidents, and transmit digital media with P2P technique. In the present, many researches focus on vehicular packet transmission and clustering to improve the efficiency of the overall system. Qadri et al. [1] presented a multi-source video streaming mechanism for VANETs and ensured its practicality and feasibility. Qian et al. [2] provided the basic design selections and rules for unmanned vehicular video streaming, and Gotta et al. [3] proposed a technique called Smart Mode to increase the Quality of Experience (QoE) and to decrease the waste of bandwidth for satellite video streaming transmission in urban vehicular environment. These applications show that the video streaming in the scope of vehicular networks is realistic and has its practical interest.

Manuscript received June 16, 2014; revised August 23, 2014. This work was financially supported in part by the National Science Council of Taiwan under contract numbers NSC 102-2511-S-259-010-MY3 and 99A058, respectively.

The authors are with the National Dong Hwa University, Taiwan (e-mail: cjhuang@mail.ndhu.edu.tw).
In future vehicular networks, the requirements of the communication between the vehicles and outside world will increase. Communication modules are expected to be embedded in vehicular entertainment system, which integrates Microsoft Silverlight streaming technique and Microsoft Expression Encoder to make Smooth Streaming effect and provide users a high-quality viewing experience.

The explosive increase in commercial usage of the Internet has led to a rapid growth in demand for video delivery technologies. Thus, efficient multimedia streaming techniques should be incorporated into the entertainment systems deployed for vehicular networks. Multimedia streaming can be operated on-demand or live. In live streaming, such as interactive video conferencing, the compressed media source needs be transmitted to multimedia players through servers and networks promptly. In on-demand streaming, all compressed media source files need be saved in the database, and the demanded files are transmitted from the database while the services are requested by the clients.

To make users receive multimedia data anywhere, many researches have been working on supporting mobility for streaming. Nasser et al. [4] adaptively compressed multimedia data to fit different multimedia wireless network conditions. Thus, more new users can be allowed to receive the services. If free bandwidth cannot support new users' requirements, call admission control (CAC) is used to drop the new requests. This research can maximize bandwidth utilization and provide users service with acceptable quality, but it is only designed for low-mobility devices. In the vehicular network environment, various traffic conditions and the clients' fast moving make multimedia transmission more complicated. Yoon et al. [5] proposed a mobility profile aided file downloading service (MoPADS) for vehicular networks. MoPADS uses drivers' mobility records to help data transmission. More specifically, MoPADS predicts users' route, moving speed, and arrival time. It also establishes a mesh network, in which each macro BS is connected with fiber backbone network and several micro BSs. The advantage of MoPADS is the cost on BS deployment will be decreased because micro BSs no longer connect to backbone network. However, service interruption may occur while a vehicle moves out of the coverage of a BS since multiple route choices will cause source transmission more complicated. Besides, vehicle-to-vehicle (V2V) transmission is not considered in this approach. Sukuvaara [6] considered both V2V and V2I (vehicle-to-infrastructure) communications to build a wireless traffic service platform (WTSP), which consists of a central traffic service server, BSs, and moving vehicles. However, this platform provides 
information that requires less bandwidth, such as accident warning and local weather report, so some streaming service was not used smoothly, and even was not available.

Aiming at seamless multimedia streaming service, Microsoft addressed Silverlight technique to provide smooth streaming effect. This technique is able to adapt to different environments automatically and make users enjoy smooth multimedia services anytime and anywhere. Smooth streaming technique can provide adequate quality of stream file and make users enjoy high-quality viewing experience according to different bandwidths. However, smooth streaming technique should be modified extensively to fit the volatile vehicular network environments.

In this work, an adaptive seamless streaming dissemination system is proposed to tackle above-mentioned challenges. The adaptive streaming system computes the parts of prefetched stream data for each user and stores them temporarily at the local server, based on current situation of the users and the environments that they are located at. Several techniques such as stream data prefetching, stream data forwarding, and adaptive dynamic decoding, were utilized for enhancing the adaptability of different users and environments and achieving the best transmission efficiency. Considering the uneven deployment of roadside base station (BS) and vehicles, the bandwidth reservation mechanism for premium user was proposed to ensure the QoS of stream data premium user received. Notably, support vector regression (SVR) is proposed and utilized to determine if a BS or a vehicle can be chosen to transfer stream data for users. SVR technique is adopted in this work because SVR has been successfully applied in many areas, such as time series prediction, Internet traffic prediction, call classification for AT\&T's natural dialog system, multi-user detection and signal recovery for a code division multiple access (CDMA) system, etc. Moreover, there are lots of solutions on VLSI chips which allow the SVR to be hardware-computed, and high-speed low cost SVR chips have been introduced recently, the implementation of SVR by hardware thus becomes feasible nowadays.

This article is organized as follows. The next section presents the multimedia streaming system. The simulation results are given in Section III, and the final section concludes the work.

\section{AN ADAPTIVE MULTIMEDIA STREAMING DiSSEMINATION SYSTEM FOR VEHICULAR NETWORKS REVIEW STAGE}

The simulated road map is first divided into several sections, and the scenario of the proposed multimedia streaming dissemination system is illustrated in Fig. 1. Based on the hierarchical framework as shown in Fig. 1, a video-on-demand (VoD) streaming dissemination server collects video, and streaming data buffers are distributed at each local server. Each road section is managed by a local server, whereas an upper-level server at a higher hierarchical level is responsible for a section with a larger scope, which is composed of several nearby road sections. According to individual user's current status, the VoD streaming dissemination server determines the amount of frames that each local server needs to prefetch and then saves data in the local server so that congestion can be effectively avoided, this architecture which shows the scalability of our proposed work.

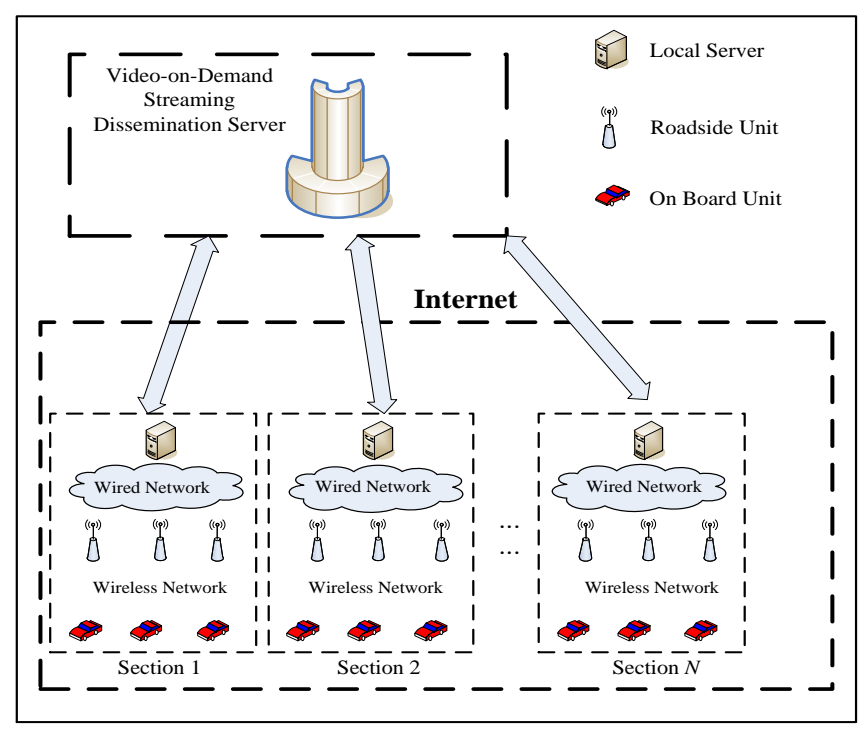

Fig. 1. Scenario of the proposed multimedia streaming dissemination system.

The proposed adaptive multimedia streaming dissemination system for vehicular networks is shown in Fig. 2. To maintain the reliable quality of the video for the viewers, three cases are considered in our proposed adaptive multimedia streaming dissemination methods. Case 1 represents the situation that the density of roadside BSs and vehicle distribution are both high; Case 2 is applied when the density of roadside BSs is sparse but that of vehicles is dense; while Case 3 is the condition that the density of roadsides BSs and vehicle distributions are both low. With the received data, the system determines each user's case to make sure the performance of video streaming service is maintained at a satisfactory level.

As shown on the left part of Fig. 3, a user first downloads and plays stream data from a roadside BS simultaneously. In Cases 1 and 2, they do not need to pre-load the stream data in advance. Although there are few roadside BSs in Case 2, the user can still receive the stream data via other vehicles as forwarding nodes. When the number of vehicles and roadside BSs is not enough to maintain the live streaming service, a pre-loading mechanism will be used to make sure that the users, especially the premium users, can still enjoy high viewing quality. The user will keep receiving the pre-loaded stream data until it leaves the range of the current BS, and then plays the pre-loaded stream data to meet a seamless live stream service. When the user enters into the range of another roadside $\mathrm{BS}$, the user will start receiving the stream data from the BS again. The above-mentioned operations of the server are shown on the right part of Fig. 2.
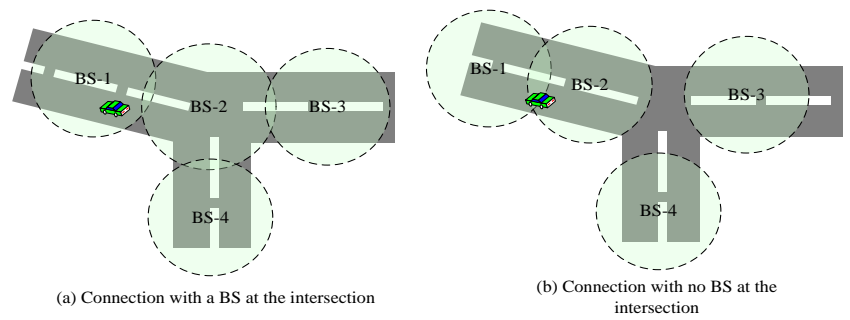

Fig. 3. The scenario of a vehicle passing through a road intersection. 


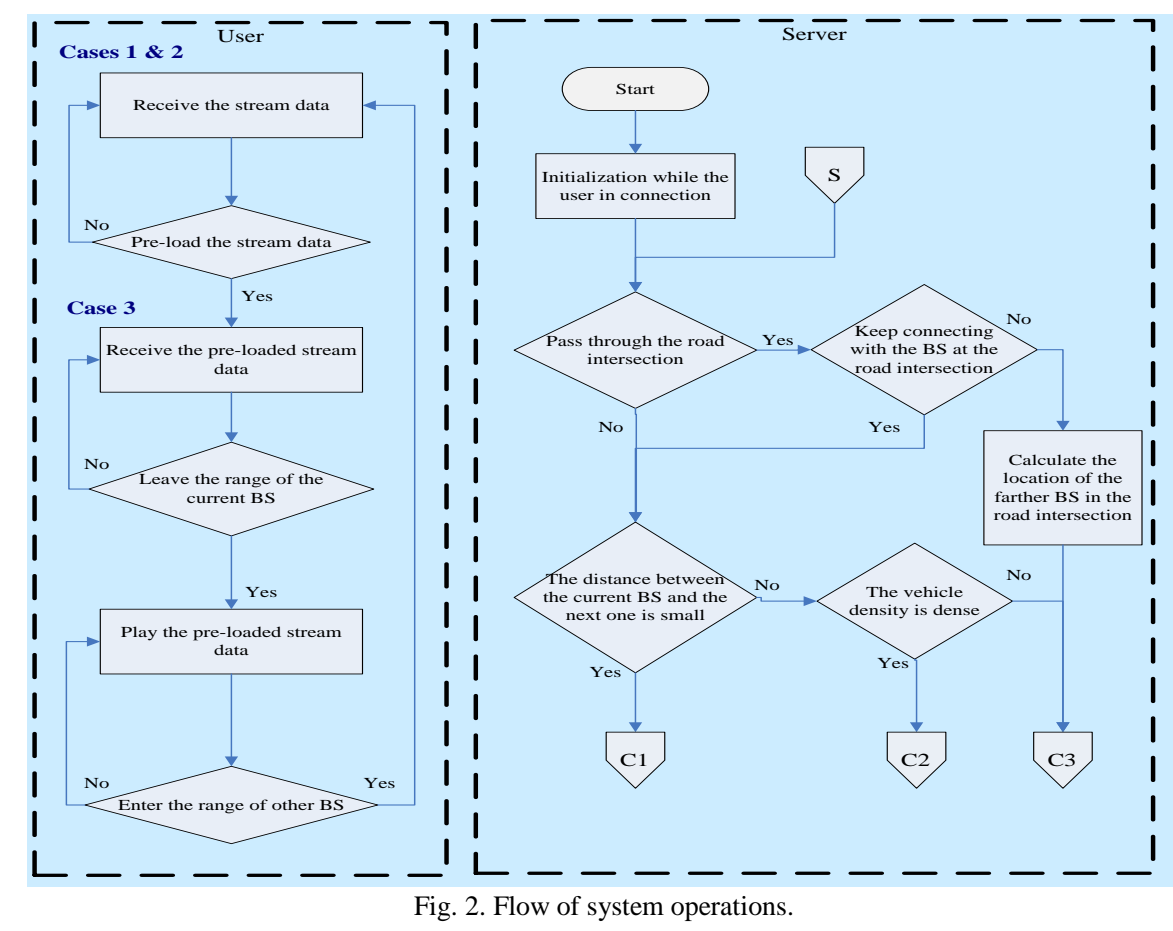

\section{A. Case 1: The Density of Roadside BSs and Vehicle Distribution Are Both High}

We can expect that the vehicle density and roadside BS distribution are both higher in populated urban areas. Although the number of roadside BSs here is much higher than that in suburbs or the normal roads, the users accessing the Internet also increase at the same time and it may cause the shortage of bandwidth and congestion. Therefore, in this case, we have to consider that the BSs have the possibility of unconnected status. Here we classify users into two types, including a premium user and a free user. In case of the shortage of the bandwidth, the free user will be degraded the quality of the streaming data to obtain more bandwidth to meet the minimum bandwidth requirements for the premium user that has higher priority.

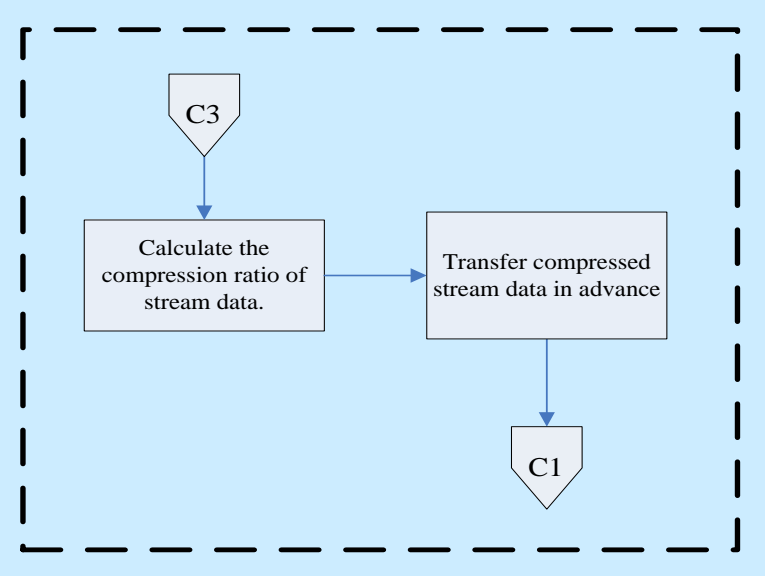

Fig. 4. The actions after Label C3.

Imagine that a vehicle is leaving the range of the current roadside BS and it will drive through an intersection as shown in Fig. 3. Fig. 3 (a) depicts that the vehicle will encounter an intersection, but no matter which direction it drives to, it always resides in the range of BS-2, and that means the server will not replace the forwarding BS.
However, Fig. 3 (b) shows that the user will leave the range of BS-2 when he/she enters into the intersection, and the stream data must be forwarded by either BS-3 or BS-4 after the vehicle passes through the intersection. The difficult part is that the server cannot determine which direction the user may drive to, so the system will select the farthest BS as the next connected BS to pre-load the necessary stream data to keep the seamless QoS according to the flowchart of Fig. 4.

As shown on the right portion of Fig. 2, when a vehicle is not going to pass through an intersection and the distance between the current BS and the next one is small, the system will take the actions after label $\mathrm{C}$, which is also the initial stage as shown in Fig. 5. If a vehicle is going to pass through an intersection, he/she can still keep connection with a BS as the case shown in Fig. 3(a), the system will take the actions after label $\mathrm{C} 1$ as well. In either of these two situations, SVR is utilized to determine whether a roadside BS is suitable for forwarding the stream data to the vehicle. If all the BSs are not appropriate to communicate with the vehicle through the judgment of the system, the server will take the actions after label $\mathrm{C} 2$, which is also the initial stage in Fig. 6. Then a vehicle will be chosen to forward the stream data after label $\mathrm{C} 2$. On the other hand, if a BS is selected to transfer stream data, the BS will allocate adequate bandwidth to prepare transferring streaming data for users.

There are three input parameters used in the SVR, including the remaining bandwidth, the number of premium users, and the number of free users in connection. Notably, inadequate bandwidth will affect the QoS of playing stream data, and the jitter or service disruption may occur. The number of premium and free users in connection would as well influence the quality of communication.

The output of the SVR is the estimated appropriateness level of a BS. The appropriateness level will be transferred to the server for further instructions. When the server initially classifies the user to take the actions after label $\mathrm{C} 1$, then it will begin to estimate the condition of the BS via the above-mentioned SVR. 


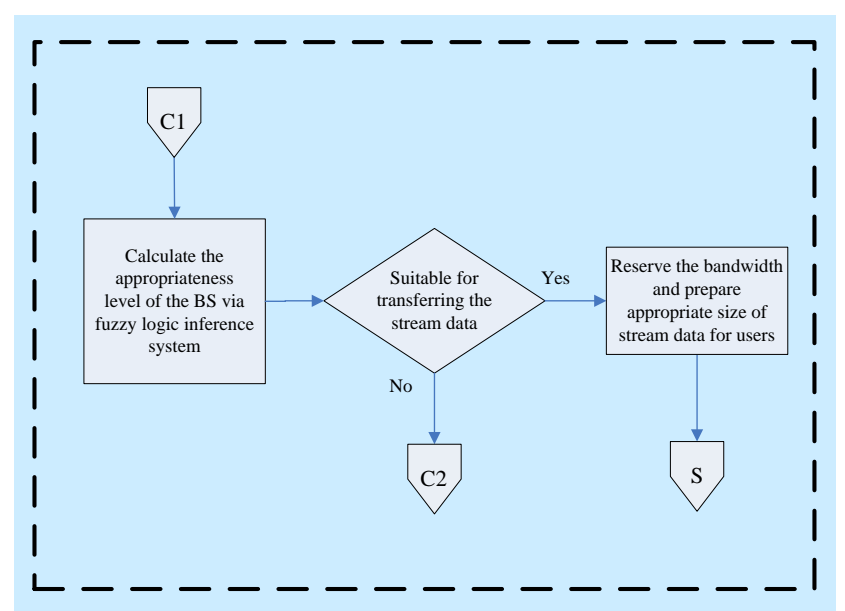

Fig. 5. The actions after Label C1.

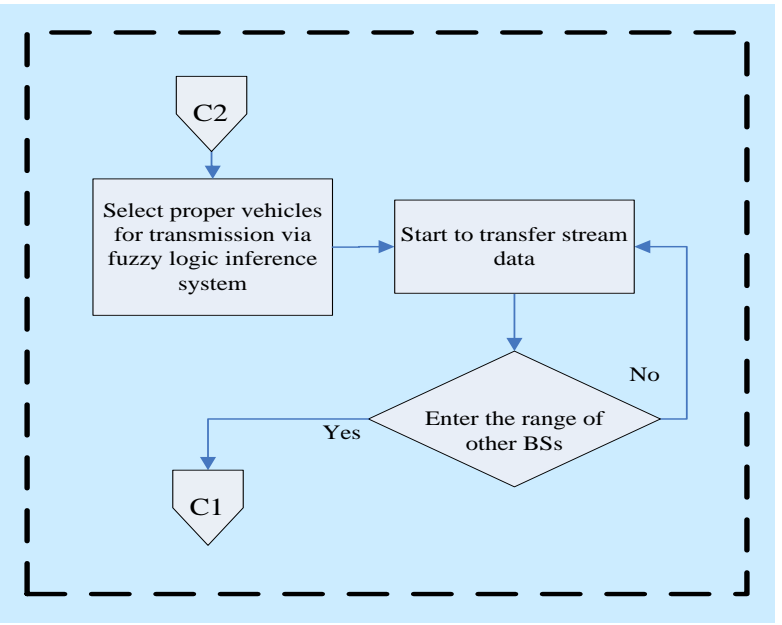

Fig. 6. The actions after Label C2.

\section{B. Case 2: Roadside BSs are Sparse but Vehicles Are Dense}

When users cannot communicate with the BS, they can also maintain the streaming service via each neighboring vehicle to transfer the stream data. In this case, our purpose is to guarantee the premium users' QoS, so the system will ask the neighboring vehicles as transmission nodes to transfer the stream data according the route built by the server. Take Fig. 7 as an example. Although the premium user in the intersection is out of the range of any roadside BS, he/she can still maintain the viewing quality of stream data via other neighboring vehicles, which are all free users.

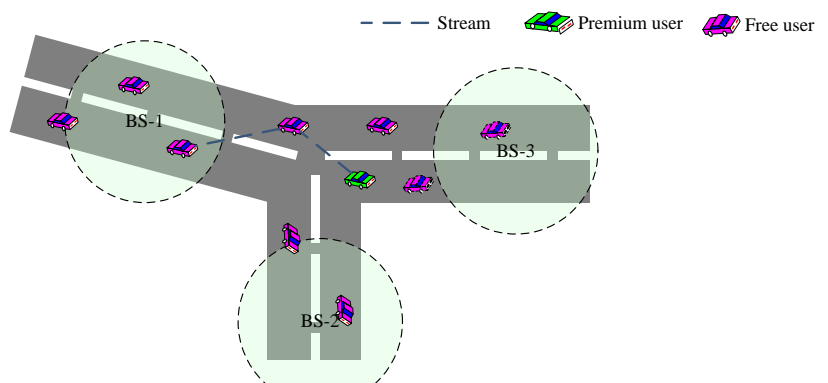

Fig. 7. An example that shows roadside BSs are sparse but the vehicle density is dense.

Parts of the right portion in Fig. 2 and Fig. 6 show the operations of the system for Case 2. In this case, the distance between the current BS and the next one is large, and the vehicle density is dense. While entering into label C2 in Fig. 6 , the system first uses SVR to select proper vehicles for transferring stream data. Next, the selected vehicles will be asked to provide bandwidth for the premium user until it enters into the transmission range of the next roadside BS. The system will then turn to the operations after label $\mathrm{C} 1$ to determine if the BS or any of other BSs is appropriate for forwarding stream data.

We adopt the SVR to determine the appropriateness level of a vehicle as a relay node. There are three input parameters used in the SVR, including the driving speed of the relay vehicle, the consumed bandwidth by the relay vehicle, and the distance from the user to the relay vehicle. Notably, the selected vehicles as the relay nodes may have the characteristics, including both the same driving direction and similar driving speed with the serviced user, which could provide stability while transferring stream data. Furthermore, the more bandwidth the vehicles have for forwarding stream data, the more success rate the user has for maintaining the viewing quality. The last but not the least, if the distance from the relay vehicle to the user is close, they will not easily leave the transmission range of each other and the relay vehicle is appropriate for relay the stream data.

The output of the SVR is the estimated appropriateness level of the vehicle as the relay node. The appropriateness level will be transferred to the server for further instructions. The fuzzy linguistic variables used for the output membership function are "low", "medium", and "high", which stand for "poor as a transmission node", "acceptable as a transmission node", and "good as a transmission node", respectively. When the server initially classifies the user as Case 2, it will begin to estimate the condition of the vehicles via the above-mentioned SVR.

\section{Case 3: The Densities of Roadsides BSs and Vehicle Distributions Are Both Low}

An example given in Fig. 8 shows that the large gap between BS-2 and BS-3 will result in a possible service disruption. To keep premium users' viewing quality, the server will ask them to activate the pre-loading mechanism to download the compressed stream data in advance.

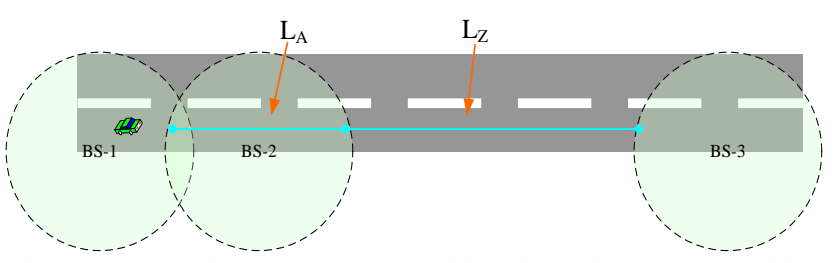

Fig. 8. An example that shows the densities of roadsides BSs and vehicle distributions are low.

Again, parts of the right portion given in Fig. 2 initiate the operations for Case 3. If a vehicle drives through an intersection and the service coverage of any BS does not cover the road intersection as the case shown in Fig. (b), the system will first calculate the location of the farther BS in the road intersection and then takes the actions after label C3, which is also the initial stage in Fig. 4. Because the user obtains stream service from neither the roadside BSs nor other nearby vehicles as transmission nodes, the server will activate the pre-loading mechanism to calculate both the distance from the current BS to the next one, and the current 
speed of the vehicle. Next the compression ratio of the stream data can be estimated with these two related data. The system will simultaneously keep playing the current stream data and pre-load the necessary steam data in advance according to the estimated compression ratio. After the user enters the coverage of any roadside BS, the server will classify the user into Case 1 and prepare the connection with the BS.

Take Fig. 8 as an example again. A vehicle is driving on the road section LA and is reaching the intersection of BS-1 and BS-2. The server is aware that this vehicle will be out of the transmission ranges of all the BSs in the following road section $L_{Z}$. Here the two parameters, $L_{A}$ and $L_{Z}$, which respectively represent the road section with connection and out of the transmission range of any BS, are used to calculate the compression ratio of the stream data. Assume that the vehicle moves with constant velocity, and the driving times on $L_{A}$ and $L_{Z}$, are directly proportional to the distance. Hence we can determine how much bandwidth we should reserve in advance while driving on $L_{Z}$ and further calculate the appropriate compression level of stream data to avoid the shortage of bandwidth in the following road section without connection of any BS.

Let $a_{L_{Z}}$ represents the required bandwidth reserved in advance on the road section $L_{z}$, where the vehicle is out of the transmission range of any $\mathrm{BS}$; and $a_{L_{A}}$ denotes the allocated bandwidth while the vehicle is on the road section $L_{A}$, during its connection with BS-2 as shown in Fig. 8. The determination of $a_{L_{Z}}$ and $a_{L_{A}}$ can be expressed below,

$$
a_{L_{Z}}=a_{i}-a_{L_{A}}
$$

And

$$
a_{L_{A}}=\left\{\begin{array}{l}
a_{n}, \quad \text { if } a_{i} \times \frac{L_{A}}{L_{A}+L_{z}}<a_{n} \\
a_{k+1}, \quad \text { if } a_{k+1} \leq a_{i} \times \frac{L_{A}}{L_{A}+L_{z}}<a_{k}
\end{array},\right.
$$

where $i$ and $a_{i}$ represent the service level of stream data and the required bandwidth on the road section $L_{A}$, respectively. Notably, the service level of the stream data and its corresponding video resolution quality used in our simulation is listed in Table I.

TABLE I: THE SERVICE LEVEL OF THE STREAM DATA AND ITS CORRESPONDING VIDEO RESOLUTION QUALITY

\begin{tabular}{|c|c|c|}
\hline $\begin{array}{c}\text { Service level of stream } \\
\text { data }\end{array}$ & $\begin{array}{c}\text { Corresponding video } \\
\text { resolution quality }\end{array}$ & $\begin{array}{c}\text { Required bandwidth } \\
\text { of each video }\end{array}$ \\
\hline$n=1$ & $1920 \times 1080$ & $500 \mathrm{~kb} / \mathrm{sec}$ \\
\hline$n=2$ & $1280 \times 720$ & $200 \mathrm{~kb} / \mathrm{sec}$ \\
\hline$n=3$ & $848 \times 480$ & $100 \mathrm{~kb} / \mathrm{sec}$ \\
\hline$n=4$ & $640 \times 480$ & $50 \mathrm{~kb} / \mathrm{sec}$ \\
\hline
\end{tabular}

Notably, our proposed scheme can adjust $a_{L_{A}}$ according to the ratio of the $L_{A}$ and $L_{Z}$. If the ratio $a_{i} \times \frac{L_{A}}{L_{A}+L_{Z}}$ is between $a_{k+1}$ and $a_{k}$ as specified at the third column in Table I, our scheme will select the lower bound of the resolution, $a_{k+1}$, to prevent the shortage of bandwidth and the possibility of service disruption. As a result, if the disconnection road section $L_{z}$ is not too long, we can still keep fine video quality with a better resolution. Otherwise, a degraded service level of stream data will be provided to users to avoid service disruption.

\section{Simulation Results}

We ran a series of simulations to verify the feasibility and effectiveness of our adaptive seamless streaming and dissemination (ASSD). The simulation parameters are shown in Table II. The size of the simulation environment is $10 \mathrm{~km} \times$ $10 \mathrm{~km}$, and the moving speed of vehicles is between $40 \mathrm{~km} / \mathrm{hr}$ and $80 \mathrm{~km} / \mathrm{hr}$. The type of the wireless networks is $\mathrm{Wi}-\mathrm{Fi}$, and the transmission range of $\mathrm{Wi}-\mathrm{Fi}$ is $80 \mathrm{~m}$. The resolution quality and the required bandwidth for each video used in our simulation is listed in Table I.

TABLE II: SIMULATION PARAMETERS

\begin{tabular}{|l|l|}
\hline Parameter & Value \\
\hline \hline Simulation area & $10 \mathrm{~km} \times 10 \mathrm{~km}$ \\
\hline Moving speed of vehicles & $40 \mathrm{~km} \sim 80 \mathrm{~km} / \mathrm{hr}$ \\
\hline Type of wireless network & $\mathrm{Wi}-\mathrm{Fi}$ \\
\hline Transmission range & $80 \mathrm{~m}$ \\
\hline Required bandwidth of videos & $500 \mathrm{~kb} / 200 \mathrm{~kb} / 100 \mathrm{~kb} / 50 \mathrm{~kb}$ \\
\hline
\end{tabular}

Two representatives of related researches in the literature, including Mobility Profile Aided File Downloading Service (MoPADS) and Wireless traffic service platform (WTSP), will be compared with our proposed work. MoPADS [5] is also an intelligent transportation system that integrates cellular infrastructure and as hoc distribution network. It uses vehicular mobility profiles not only to provide quality yet affordable services but also to predict the vehicle's possible route, velocity, and the required time to get to some specific place in the future. WTSP [6] considers two types of communications, including vehicle-to-vehicle and vehicle-to-infrastructure, to construct a wireless service platform based on the traffic service central unit, the base station network with traffic service base stations, and mobile end users with ad-hoc connectivity and backbone network connectivity. It provides the real-time service to the mobile user of less bandwidth requirement.

Throughput, packet loss ratio, and service disruption time are adopted as performance metrics in this work. Throughput represents overall multimedia data transmission capacity, which includes the data received from adaptive streaming server and the data shared with one another through BSs bandwidth sharing mechanism. Packet loss ratio stands for the percentage that packets get lost to produce errors in the transmission process. Service disruption time is defined as the period of time when the vehicle cannot maintain active connection with the current BS until it connects with a new serving BS link and restarts sending and receiving packets.

Fig. 9 illustrates the packet loss ratio on the condition of different numbers of vehicles among the three schemes. As a whole, our proposed ASSD scheme achieves better performance in the packet loss ratio than the other. Our ASSD scheme is able to manage different conditions and guarantee QoS according to different types of traffic environment. For example, if the density of vehicle distribution is low, the proposed prefetch mechanism can 
avoid the problems of transmitted streaming data interruption and delay due to the inadequate hardware resource. On the other hand, if vehicle density is high, we select relay vehicles to transmit stream data to make sure the QoS for users. However, in the architecture of MoPADS, only a few server stations are connected to the backbone via high-bandwidth wires, and the others small BSs access the networks by sharing the bandwidth of large BSs. When the traffic flow increases, the shortage of bandwidth will occur, and thus the packet loss ratio will increase. As for WTSP, it provides the real-time service with a lower requirement of bandwidth. The packet loss becomes higher when the bandwidth demand of the real-time service gets larger. WTSP can alleviate the deterioration of the packet loss ratio by adopting vehicle-to-vehicle communication to transfer stream data while the density of the vehicle is high.

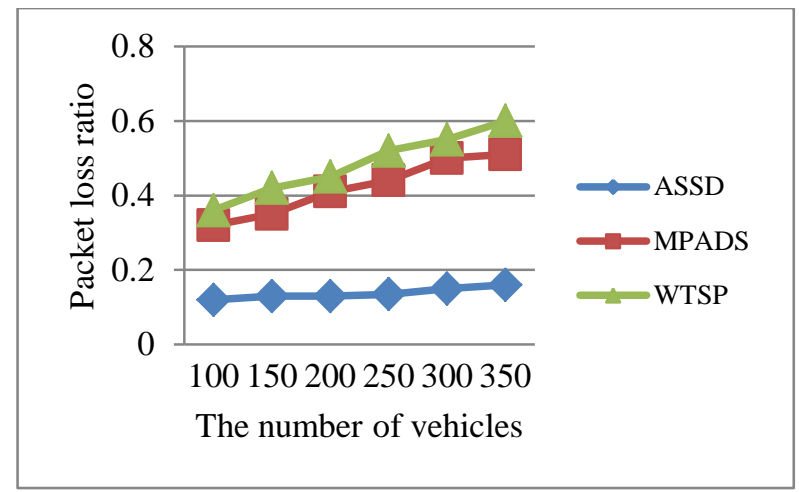

Fig. 9. Comparison of packet loss ratio among the three algorithms.

Fig. 10 illustrates the normalized service disruption time on the condition of different numbers of vehicles among the three schemes. Our proposed ASSD scheme will be well controlled since we considered the distribution of vehicles and BSs. If the density of vehicles is high, the system will transfer stream data via other selected vehicles according to the suitable distance and velocity among the vehicles. But if the density of vehicles is too low to transfer the data, the prefetching mechanism will start to make sure uninterrupted transferring of stream data to maintain the QoS. MoPADS scheme does not consider vehicle-to-vehicle communication, so when the vehicle drives away the coverage of the BS, or faces the shortage of bandwidth provided by the small BS, the service disruption time will be obviously increasing when the number of vehicles and the requirement of bandwidth increase. On the other hand, since WTSP scheme only has vehicle-to-vehicle communication but no service prefetching mechanism to transfer stream data, the service disruption time does not significantly get worse when the number of vehicles increases.

Fig. 11 illustrates the throughput on the condition of different numbers of vehicles among the three algorithms. Since some techniques such as stream data prefetching, stream data forwarding, and adaptive dynamic decoding, were applied to strengthen the adaptability of different users and environments, our proposed ASSD scheme can maximize data throughput among the three schemes. In the MoPADS scheme, small BSs may face the shortage of bandwidth to transfer stream data, but once the vehicles drive again to the coverage of the small BSs, it still can complete the packet transmission. In the WTSP scheme, although it constructs the platform based on V2V and V2I communications, it provides the real-time service to the mobile user of less bandwidth requirement. Hence, the mobile user with high bandwidth requirement will not be suitable for this scheme.

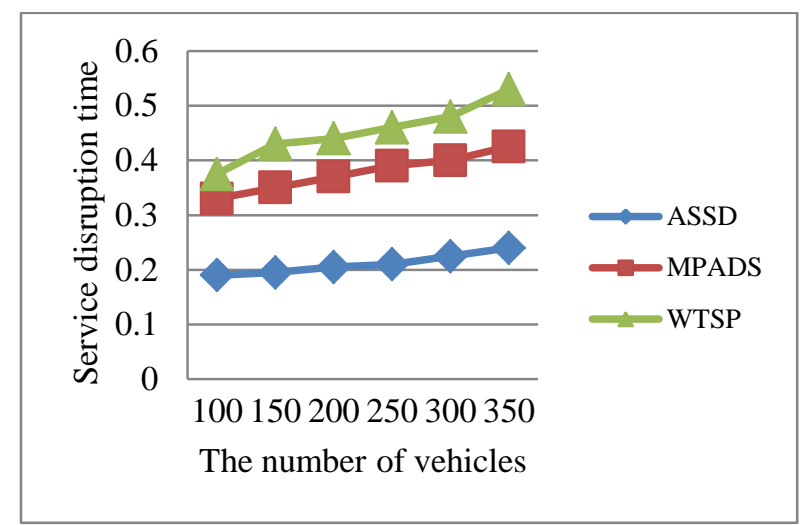

Fig. 10. Comparison of normalized service disruption time among the three algorithms.

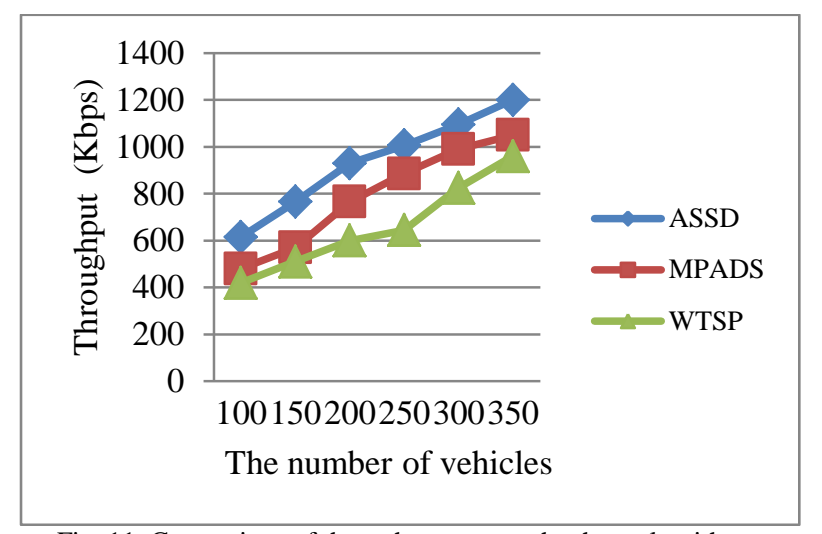

Fig. 11. Comparison of throughput among the three algorithms.

\section{CONCLUSION}

With rapid development of wireless technologies, the needs for the stream service on mobile devices are increasing nowadays. Playing live stream videos in the vehicle thus becomes possible via VANET and roadside BSs. However, there are still some difficulties to be overcome, such as the uneven distribution of roadside BSs, high mobility of vehicles, and volatile network environment. In this work, an adaptive seamless streaming dissemination system in vehicular networks is proposed to solve the above-mentioned problems. Three cases are designed to deal with different kinds of network environment according to the number of vehicles and roadside BSs. In the case of facing few roadside $\mathrm{BSs}$, the user can still receive the stream data via other vehicles as forwarding nodes. When the number of vehicles and roadside BSs is not enough to maintain the live streaming service, a pre-loading mechanism will be used to make the premium users enjoy satisfying viewing quality to meet a seamless live stream service. Comparing our simulation model with the two latest frameworks, including MoPADS and WTSP, the simulation results show that our mechanism achieves better performance in terms of packet loss ratio and service disruption ratio. The capability of self-adaption in volatile vehicular environment assists in the effectiveness and practicability of our proposed approach. 


\section{REFERENCES}

[1] N. N. Qadri, M. Fleury, M. Altaf, and M. Ghanbari, "Multi-source video streaming in a wireless vehicular ad hoc network," IET Communications, vol. 4, no. 11, pp. 1300-1311, July 2010.

[2] H. X. Qian, J. S. Huang, and L. L. Ma, "The design challenges for unmanned vehicular video streaming," in Proc. 2011 IEEE International Conference on Vehicular Electronics and Safety, pp. 19-24, July 2011.

[3] A. Gotta, E. Ferro, and F. Potorti, "Quality of experience in satellite video streaming transmissions in urban vehicular environment," in Proc. 2009 IWSSC International Workshop on Satellite and Space Communications, Sept. 2009, pp. 18-22.

[4] N. Nasser, "Service adaptability in multimedia wireless networks," IEEE Transactions on Multimedia, vol. 11, no. 4, pp. 786-792, Jun. 2009.

[5] S. Yoon, D. T. Ha, H. Q. Ngo, and C. Qiao, "MoPADS: A mobility profile aided file downloading service in vehicular networks," IEEE Transactions on Vehicular Technology, vol. 58, no. 9, pp. 5235-5246, Nov. 2009.

[6] T. Sukuvaara and P. Nurmi, "Wireless traffic service platform for combined vehicle-to-vehicle and vehicle-to-infrastructure communications," IEEE Wireless Communications, vol. 16, no. 6, pp. 54-61, Dec. 2009.

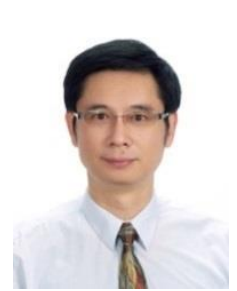

Chenn-Jung Huang received the B.S. degree in electrical engineering from National Taiwan University, Taiwan and the M.S. degree in computer science from University of Southern California, Los Angeles, in 1984 and 1987, respectively. He received the Ph.D. degree in electrical engineering from National Sun Yat-Sen University, Taiwan in 2000. He is currently a professor in the Department of Computer Science \& Information Engineering, National Dong Hwa University, Taiwan. His research interests include computer communication networks, data mining, and diagnosis agent for e-learning.

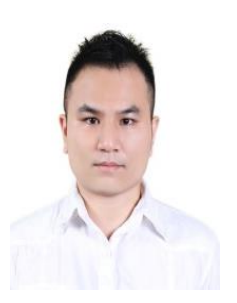

Kai-Wen Hu is pursuing his doctoral degree at the Department of Electrical Engineering, National Dong Hwa University, Taiwan. His research interests include computer communication networks, data mining, applications of machine learning techniques and e-learning.

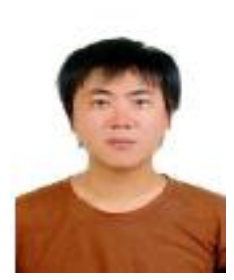

Heng-Ming Chen is pursuing his doctoral degree at the Department of Electrical Engineering, National Dong Hwa University, Taiwan. His research interests include computer communication networks, data mining, applications of machine learning techniques and e-learning.

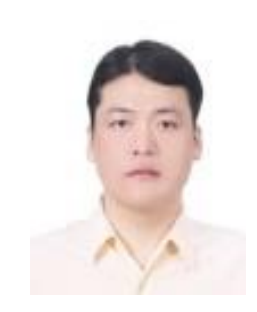

Yu-Wu Wang is pursuing his doctoral degree at the Department of Computer Science and Information Engineering, National Dong Hwa University, Taiwan. His research interests include computer communication networks, data mining and applications of machine learning techniques.

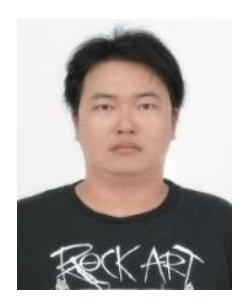

Jui-Jiun Jian is pursuing his master's degree at the Institute of Electronics Engineering, National Taiwan University, Taiwan. His research interests include computer communication networks, data mining and applications of machine learning techniques.

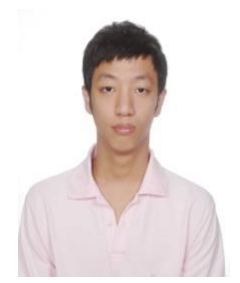

Sheng-Yuan Chien is pursuing his master's degree at the Department of Computer Science and Information Engineering, National Dong Hwa University, Taiwan. His research interests include computer communication networks, data mining and applications of machine learning techniques. 
Image Processing and Pattern Recognition 
